\title{
Eva Borwowska de Mikusinski, H. J. Eysenck y los estudios científicos de la personalidad en Argentina
}

\section{Eva Borkowska de Mikusinski, H. J. Eysenck e o estudo científico da personalidade na Argentina}

\author{
Eva Borkowska de Mikusinski, H. J. Eysenck and the scientific \\ studies of personality in Argentina
}

\author{
María Andrea Piñeda* \\ Universidad Nacional de San Luis - UNSL, San Luis, Argentina
}

\begin{abstract}
RESUMEN
Presentaremos una historia local de perspectiva policéntrica sobre Eva Borkowska de Mikusinski, una mujer polaca que migró a la Argentina tras la segunda guerra mundial, y que se convirtió en una de las primeras psicólogas graduadas de la región. Exploraremos algunas motivaciones que la impulsaron como mujer, en su vida familiar y su carrera científica, donde el hallazgo y el encuentro con $\mathrm{H}$. J. Eysenck imprimieron un rumbo decisivo para ella y su comunidad académica. Relataremos circunstancias que llevaron a esta psicóloga a buscar respuestas científicas sobre la motivación del comportamiento humano, y a convertirse en referente pionera de un programa de investigación y de formación de psicólogos basado en el modelo de Eysenck, en un contexto nacional de hegemonía psicoanalítica. Explicaremos las condiciones del sistema universitario y científico argentino que, por un lado, le permitieron a Eva desarrollar su profesión y, por otro lado, limitaron el alcance y la visibilidad de su obra.
\end{abstract}

Palabras clave: historia, mujeres, rasgos de personalidad, Eysenck, Argentina.

\section{RESUMO}

O texto apresenta uma abordagem policêntrica relativa a uma história local sobre Eva Borkowska de Mikusinski.Polonesa que migrou para a Argentina após a Segunda Guerra Mundial, tornou-se uma das primeiras psicólogas graduadas na região. Algumas de suas motivações são exploradas ao mostrar certos dilemas entre sua vida familiar e profissional.Descrevem-se as circunstâncias em que conheceu $\mathrm{H}$. J. Eysenck, explicando como esse fato a levou a novos projetos que propiciaram uma mudança histórica a sua comunidade acadêmica. Sua pesquisa científica sobre as motivações do comportamento humano permitiu que se tornasse uma referência local pioneira no modelo de Eysenck, no qual ela baseou o treinamento de psicólogos e um programa de pesquisa, apesar da cultura hegemônica psicanalítica. As condições da universidade argentina e do sistema científico são descritas, com o objetivo de explicar, por um lado, como elas permitiram que ela desenvolvesse sua profissão, mas também como, por outro lado, inibiram o escopo e incidência de seu trabalho. 
Palavras-chave: história, mulheres, traços de personalidade, Eysenck, Argentina.

\begin{abstract}
A policentric approach in a local history about Eva Borkowska de Mikusinski is presented. She is a polish woman who migrated to Argentina after the Second World War, becoming into one of the first graduated psychologist in the region. Some of the motivations of this woman are explored while showing certain dilemmas between her familiar and professional life. The circumstances when she met $\mathrm{H}$. J. Eysenck are described, explaining how this fact drove her to new projects that led her academic community to a historical change. Her scientific research about human behaviour's motivations let her become into a pioneer local reference on Eysenck's model, on which she based a psychologists' training plan and a research program, in despite of psychoanalytic hegemonic culture. Conditions of the Argentine university and scientific system are described, aiming to explain, how they let her develop her profession, but also how they inhibited the scope and incidence of her work.
\end{abstract}

Keywords: history, women, personality traits, Eysenck, Argentina.

\title{
1 Introdução
}

¿Qué motiva a una mujer a aplazar sus aspiraciones, nacidas en la niñez, de ser científica; a sobrevivir en un campo de concentración de la gélida estepa soviética; a poner en riesgo su vida como oficial en una guerra; a migrar a un país distante en kilómetros y en cultura; a iniciar una carrera académica que aún no goza de suficiente reconocimiento social; a aventurarse a ser pionera de caminos que no transitará la mayoría, y justo cuando se están realizando sus aspiraciones, qué motiva a una mujer a dejarlo todo para volver a empezar?

Presentaremos una historia local de perspectiva policéntrica (Brock, 2014) sobre Eva Borkowska de Mikusinski (1921-), una mujer polaca que migró a la Argentina tras la segunda guerra mundial, y que se convirtió en una de las primeras psicólogas graduadas de la región. Nuestro objetivo es rescatar del olvido la figura de esta mujer, demostrando que fue pionera en la introducción de las teorías de Eysenck en Argentina desde las que realizó aportes sistemáticos a los estudios científicos de la personalidad en el país.

Es conocido que, en Argentina, desde la organización de las carreras de psicología el psicoanálisis dominó la formación delos psicólogos (Dagfal, 2018), registrándose mayor apertura a otros modelos recién a principios de 1980 (Korman, Viotti, \& Garay, 2015).Sin embargo, suele pasar inadvertido que esta impronta en la formación no fue absoluta. Las primeras cinco carreras en universidades nacionales argentinas se crearon entre 1955 y 1958. Una de ellas, la de San Luis, inauguraba su curso introductorio de 1958 presentando la psicología científica desde la óptica de Hans Eysenck, un conocido 
detractor del psicoanálisis. Este hecho, muestra indicios de una matriz de formación diferente ala dominante en el paísy de una recepción de la obra del autor europeo más temprana que en el resto de Argentina. Pone en discusión literatura existente sobre la recepción de las teorías eysenckianas en los estudios de la personalidad de Sudamérica (Flores-Mendoza et al., 2016; Korman, et al., 2015; Schmidt, De Costa Oliván, Firpo, Vion, \& Casellans, 2008). Por último, evidencia un panorama complementario sobre los estudios factoriales de la personalidad que dialogan con el modelo de Eysenck, como los de L. Thurstone (1887-1955), o R. B. Catell (1905-1998) que, si bien han sido considerados modelos competidores, también han sido reconocidos como complementarios por sus mismos autores (Boyle, et al., 2016). En Argentina, el referente más renombrado en estudios desde la perspectiva de Thurstone y Cattell, ha sido Horacio Rimoldi (1913-2006). Él introdujo estos modelos en diferentes grupos de trabajo que formó: en Mendoza con Nuria Cortada (1921-2013) (Cortada, 2006), en Córdoba vinculado al Centro de Investigaciones Acústicas y Luminotécnicas (Ferrero, Iemme Khorry, Todisco y Scherman, 2016) y la Universidad Nacional de Córdoba con Hermelinda Fogliatto (1925-2003) y, más tarde, en Buenos Aires en el Centro Interdisciplinario de Investigaciones en Psicología Matemática y Experimental. Asimismo, durante la década de 1960, Rimoldi recibió en su laboratorio de Psicología Experimental en la Universidad de Loyola (Chicago) a numerosos investigadores de todo el mundo, algunos argentinos, que realizaron tesis doctorales siguiendo los modelos de Thurstone y Cattell que él utilizaba (Rimoldi, 1995; Carpintero, 2006).

¿Qué condiciones hicieron posible esta temprana recepción de Eysenck en San Luis? Este suceso no se puede analizar sin hacer referencia a los esfuerzos pioneros de Eva B. de Mikusinski. Sacando a la luz su historia, este trabajo pretende aportar a la línea de investigación sobre mujeres pioneras olvidadas en la historia de la psicología (Furumoto, 1992; Giménez, 2007; Jacó-Vilela, DeganiCarneiro, y Messias, 2012; Milar, 2000; Ostrovsky, 2008; Radtke, Hunter y Stam, 2000; Winckler Müller \& Reyes, 2015), desde una perspectiva que analiza los modelos de auspicios personales (Sprung, \& Sprung, 1996) y las condiciones de la política pública (French, 1988) para la integración de mujeres en la actividad científica y académica en psicología. Justamente por eso, esta tarea no resulta sencilla. Parte de esa complejidad radica en bosquejar en pocos trazos el encuentro de Eva con el conocimiento y las instituciones científicas, un campo en el que Eysenck cobraría un significado especial, contribuyendo a motivar sus metas y a forjar su identidad profesional. El otro desafío, consiste en hilar la trama en la que sus motivaciones personales se conjugaron con las de sus pares para 
impulsar un cambio histórico en una comunidad de psicólogos. En ese sentido, este trabajo también se inscribe entre los estudios sobre historia de la investigación en psicología en Argentina, especialmente aquellos trabajos que han analizado políticas académicas y científicas de la segunda mitad del siglo XX (Piñeda, 2012, Piñeda y Scherman, 2016a), así como las principales comunidades de investigadores de la región (Ardila, 1979, Papini y Mustaca, 1979, Mustaca, 2006, Piñeda y Jacó-Vilela, 2014), entre las que se cuenta la de San Luis (Klappenbach, 2011; Piñeda, 2010).

Desde esta triple perspectiva histórica sobre: mujeres pioneras en la psicología, la recepción de Eysenck y de modelos factoriales en los estudios científicos de la personalidad, y la investigación en Psicología en Argentina a mediados del siglo XX, rescataremos la figura de Eva B. de Mikusisnki, como mujer científica, demostrando que fue introductora de las teorías de Eysenck en este país y que, desde dicho marco, realizó aportes sistemáticos a los estudios científicos de la personalidad en ese contexto. Nos interesa explorar algunas motivaciones que impulsaron a Eva como mujer en su acceso a la carrera científica, conjugando dos planos no exentos de tensiones: su vida familiar y su carrera. De modo particular, señalaremos cómo el hallazgo y el encuentro con $\mathrm{H}$. J. Eysenck imprimieron un rumbo decisivo en su proyecto de vida, y en el desarrollo de su comunidad académica. Describiremos los auspicios que la promovieron para integrarse a la comunidad académica nacional e internacional, y para convertirse en referente de un programa de investigación y de formación de psicólogos basado en el modelo de Eysenck. Asimismo, explicaremos las condiciones del sistema universitario y científico argentino que le otorgó oportunidades y, al mismo tiempo, limitó su desarrollo.

Para ello, se ha recurrido a numerosas fuentes primarias. En primer lugar, se ha entrevistado a la Dra. Eva B. de Mikusinski, registrando en formato digital más de diez horas de entrevista que abordan su vida desde la infancia hasta el retiro de su carrera científica. Se ha accedido a su archivo personal analizando documentos como: el legajo en la Universidad Nacional de Cuyo (UNCy), en la Universidad Nacional de San Luis (UNSL) y en el Consejo Nacional de Investigaciones Científicas y Técnicas (CONICET); epístolas (Ej. a Hans Eysenck, Robert Kastembaun y Héctor Fernández Álvarez) y fotografías familiares, de su carrera militar y científica. Por otra parte, se han analizado materiales originales del Archivo y Centro de Documentación Plácido Horas de la Facultad de Psicología de la UNSL. Entre ellos, documentos institucionales: Libro de Egresados, memorias anuales del Instituto de Investigaciones Psicopedagógicas (UNCy) y de la Secretaría de Ciencia y Técnica (UNSL), designaciones docentes, planes de estudios, programas de cursos dictados por los principales mentores de Mikusinski en San Luis: Plácido Horas 
(Psicología General) y Francisco Murat (Metodología de la Investigación) de los que se realizó análisis de contenidos y de referencias bibliográficas. También se accedió a todas las tesis de grado y de posgrado realizadas en la carrera de psicología de San Luis entre 1958 y 1983, entre las que se contaron el trabajo final de grado y de doctorado de Mikusinski - de los que se realizó análisis de contenido y de referencias bibliográficas-, y el centenar de tesis que ella dirigió - de las que se procedió a clasificar por temática. Asimismo, se analizaron las notas e informes de investigación y de dirección de becarios en proyectos dirigidos por Mikusinski en la UNCy, UNSL y CONICET. Se revisó el Acta Fundacional de la Asociación Argentina de Ciencias del Comportamiento (AACC) de la que Mikusinski fue miembro fundador. Finalmente, se realizó un rastreo y análisis exhaustivo de las publicaciones de Mikusinski y colaboradores en revistas científicas.

\section{$2 \mathrm{H}$. J. Eysenck en el contexto latinoamericano}

Trasel centenario del nacimiento y dos décadas del fallecimiento de Eysenck (1916-1997), aún se lo considera uno de los psicólogos más influyentes del siglo XX (Caballo, 1997; Corr, 2016; Diener, Oishi, \& Park, 2014). Sus aportes sobre las bases biológicas de la personalidad continúan provocando fértiles líneas de investigación en el siglo XXI (Mitchell \& Kumari, 2016). El joven alemán que migró a Inglaterra alejándose del nazismo, casi por casualidad estudió psicología, convirtiéndose en el último de los psicólogos sistémicos del siglo XX, y entre los primeros en proponer una psicología urbana, enfocada a problemas específicos de la vida cotidiana (Pinillos, 1997). $\mathrm{Ha}$ contribuido al estudio científico de la personalidad y a la psicoterapia (Arias Gallego, 2012; Eysenck, 2016), como también ha estimulado estudios y debates en campos a le daños: psicología política, criminología, ciencias biomédicas, administración, economía, derecho y educación, en los que dirigió gran cantidad de tesis doctorales (Carpintero, Tortosa, \& Sanchis-Aldas, 1997).

De su prolífica obra, destacaremos Dimensions of Personality (1947), su primer intento de construir un sistema descriptivo de la personalidad que alcanzó una rápida difusión. Numerosas publicaciones contribuyeron a dar cuerpo a este proyecto, entre las más conocidas, The Scientific Study of Personality (1952). Allí se aprecia el afán de Eysenck por investigar el comportamiento desde las bases biológicas de la personalidad (Gottfredson, 2016), procurando describir operacionalmente sus dimensiones con procedimientos experimentales, psicométricos y psicofisiológicos.

Por otra parte, en una época en la que destacamos la comunicación pública de las ciencias para el empoderamiento que las sociedades 
puedan hacer de estas, subrayamos los esfuerzos de Eysenck por dirigirse al lector sin conocimientos previos de psicología o estadística, con la convicción de aportar a las decisiones sociales basadas en el conocimiento científico (Carpintero et. al, 1997). Entre sus divulgados libros de bolsillo, mencionamos Uses and Abuses of Psychology (1953).

Se ha señalado el impacto que Eysenckha tenido en Latinoamérica desde mediados de 1960, gracias a las traducciones españolas, mexicanas y argentinas de sus textos y cuestionarios que empezaron a editarse desde fines de 1950 (Flores-Mendoza et. al., 2016). La Revista Latinoamericana de Psicología también visibilizó su obra en nuestra cultura académica (Eysenck, 1977). Pero su mayor popularización en el campo de la terapia del comportamiento se logró con sus tres visitas a Latinoamérica: 1981 en Argentina; 1984 en México y 1993 en Colombia (Korman, et al., 2015).

Respecto del ámbito académico argentino, se han discutido falencias en la formación de grado y de posgrado de los psicólogos en el campo de la evaluación científica de la personalidad, así como en las condiciones de infraestructura en centros universitarios, que han limitado la enseñanza de la obra de Eysenck y su incidencia en la investigación. En ese sentido, se ha advertido que, los pocos trabajos realizados en el país, desde esta perspectiva, dependieron de esfuerzos aislados más que de programas sistemáticos (Schmidt, et al., 2008). No obstante, se han valorado los aportes de Alicia Omar (1988), quien desde Rosario ha contribuido con las primeras adaptaciones en Argentina y posiblemente de Latinoamérica (FloresMendoza et al., 2016). Asimismo, se ha registrado alguna incidencia del modelo de Eysenck en la Facultad de Psicología de la Universidad de Buenos Aires: en cursos de Metodología de la Investigación, Psicometría o Psicoterapias dictados por María del Rosario Lores Arnaiz, Alba Mustacay Eduardo Keegan, y en líneas de investigación conducidas por Lores Arnaiz y Schmidt. Finalmente, también se registró esta incidencia en la tarea docente y de investigación de Claribel Morales de Barbenza en la UNSL (Schmidt et. al., 2008).Sin embargo, hemos recogido indicios de una recepción más temprana de la obra de Eysenck en San Luis y de que la misma ha sido significativa en la constitución de la cultura científica de una comunidad de psicólogos comportamentales (Piñeda, 2010; Piñeda y Jacó-Vilela, 2014), con la que algunas líneas investigativas eysenkianas del país tendrían su deuda.

Repararemos en el interés por Eysenck de parte de la naciente psicología de San Luis durante la década de 1950. En particular, destacaremos el papel que jugó Eva Borkowska de Mikusinski, la primera psicóloga graduada en San Luis, en la promoción de investigación y formación de psicólogos bajo el modelo eysenckiano. 


\section{Eva Borkowska de Mikusinski y el conocimiento científico}

Eva Borkowska nació en 1921 en Lvov, Polonia. Desde pequeña manifestó inquietudes por el conocimiento científico en el campo humano y social. Este apetito fue habilitado y nutrido por su padre y su abuelo que la instruyeron en cultura, artes y ciencias, estimulando lecturas y relatando sus viajes (Mikusinski, comunicación personal, 13 de abril de 2018). También contribuyeron sus estudios humanistas y de idiomas en el Colegio de las Hermanas de Notre Dame. En su preadolescencia accedió a la "Introducción al Psicoanálisis" de Freud, y al manual Esquema de psicología de Wladyslaw Witwicki (18781948), considerado padre de la psicología polaca (Citlak, 2016). Este último objeto, acaso se haya transformado en símbolo de las raíces de Eva, así como de su proyecto de vida, aspiraciones y legado. Tras los sucesos bélicos de 1939, ella se aferró al manual que leyó a los diez años, prefigurando una suerte de profecía autocumplida. La joven que soñaba con estudiar en la universidad -en esa época, derecho o filología-, no vería desilusionada sus expectativas aun cuando le negaban el acceso por ser hija de burgueses (Mikusinski, 1996). La dramática guerra tampoco la detendría.

La suerte de toda su familia corrió grandes riesgos tras desatarse la Segunda Guerra Mundial. Polonia sufrió la invasión nazi, y luego, del ejército soviético. El padre de Eva, que servía al ejército polaco, se salvó de la masacre de Katyn, en la que por orden de Stalin se fusilaron más de 4000 oficiales polacos. Sin embargo, fue tomado prisionero mientras Eva, su madre y hermano fueron deportados a la República Soviética de Kazajistan durante dos años. Tras perder contacto con el padre y vivir estremecedoras experiencias de supervivencia, en 1942 fueron liberados y la familia logró reintegrarse. Eva se enlistó en el Servicio Militar bajo comando británico, llegando a ser oficial. Se desplazó desde Egipto y Palestina hasta Inglaterra e Irlanda. En 1945, poco antes de concluir la guerra, fue trasladada a Francia, donde conoció a su futuro esposo. Pudo aplicar para el ingreso a universidades británicas y norteamericanas, pero priorizó el proyecto familiar de migrar a la Argentina donde parecían abrirse mejores oportunidades laborales para su marido (Mikusinski, 1996). Antes por ser "hija de", ahora por ser "esposa de", nuevamente sus aspiraciones académicas fueron aplazadas por las familiares, y esta no sería su última encrucijada.

Los Mikusinski se vieron alentados a probar suerte en Argentina que comenzaba un proceso de modernización e industrialización durante los dos primeros gobiernos de Juan Domingo Perón entre 1945 y 1955. San Luis fue elegida para establecerse porque se empleaba mano de obra para la construcción de represas, bajo el gobierno de Ricardo Zavala Ortiz. Desde el censo nacional de 1947, se registró un sostenido aumento de la participación femenina en la actividad 
económica, concentrándose el $68 \%$ en el sector terciario, que incluye servicios (como educación, salud y administración). Actividades como docencia o enfermería, se consideraban "feminizadas" en tanto se asociaban a roles domésticos otrora reservados a las mujeres, como cuidar y consolar (Falcone, 2015). En ese contexto, mientras el esposo de Eva encontró trabajo como electromecánico, ella se empleó como traductora y docente de inglés.

En San Luis, pequeña ciudad del centro de Argentina, se estaban configurando nuevos escenarios culturales con la apertura de los estudios universitarios. En 1939 se creó la Universidad Nacional de Cuyocon gobierno en Mendoza, de la que dependían Institutos en San Juan y San Luis. El Instituto Nacional de Profesorado de San Luis fue organizado en 1940, y reorganizado en 1941 como Instituto Pedagógico para jerarquizar la formación docente (Klappenbach, Marincevic, Arias \& Montoya, 1995). Pronto este objetivo evolucionó hacia la formación profesional y el desarrollo científico.

En dicho marco, en 1943 llegó a San Luis Plácido Horas recientemente graduado en la Universidad de Buenos Aires. El joven profesor de filosofía, sintetizaba las tradiciones filosófica y científica representadas por sus docentes de psicología, los célebres Coriolano Alberini (1886-1960)y Enrique Mouchet (1886-1977). Para Horas, la psicología era la ciencia del hombre total, el estudio de la personalidad y del comportamiento en todas sus dimensiones, rechazando cualquier enfoque reduccionista y dogmático. Sostuvo un integracionismo teórico y metodológico, introduciendo en la psicología sanluiseña tanto conductismo, humanismo, reflexología como psicoanálisis (Piñeda, 2007).

En 1946, el Instituto fue elevado al rango de Facultad de Ciencias. Contaba con un Instituto de Investigaciones Pedagógicas, dirigido por Horas, y desde 1949, un Profesorado en Pedagogía y Filosofía. La psicología fue ganando terreno con una Especialización en Psicología en 1953, la reorganización en Instituto de Investigaciones Psicopedagógicas en 1956, y la creación de la carrera de psicología en 1958 (Klappenbach et al., 1995). En ese escenario, en 1949 Eva ingresó a estudiar en la universidad, siendo Horas su docente de Psicología General y de Psicología del Niño y del Adolescente.

Simultáneamente, Eva B. de Mikusinski -nombre bajo el que firmaba ya casada y en tierras argentinas-trabajaba como bibliotecaria y traductora de la Asesoría Técnica del Ministerio de Educación de la Provincia (Mikusinski, 1996) que había celebrado un convenio con la Facultad de Ciencias. En virtud de éste, y en pleno auge de la psicotecnia, en el Instituto se realizaba capacitación para los docentes y evaluación psicométrica en escuelas (Piñeda, 2010). Una reorganización de las instituciones estatales tras el golpe de estado de 1955 reasignó a Eva al Instituto de Investigaciones donde, bajo la dirección de Horas, se inició como investigadora y auxiliar docente. 
Al año siguiente se abrió la carrera de psicología en San Luis. Eva se contó entre sus primeras alumnas. Ese año, tuvo a su única hija. Poco después, quedó a cargo de su madre e hija, debido al distanciamiento del matrimonio seguido de viudez (Mikusinski, comunicación personal, 13 de abril de 2018).

Acaso estas circunstancias alentaron sus motivaciones de logro profesional. Su empeño la transformó en 1963 en la primera licenciada en psicología en San Luis (Universidad Nacional de Cuyo, n.d.). Su sufrida vida no desalentó sus objetivos, sino que fue terreno fértil para verlos fructificar. Esta posición existencial, se entreveía en sus indagaciones científicas ¿Existen rasgos de personalidad que inciden para que, dadas las mismas aptitudes y aspiraciones en diferentes individuos, algunos determinen su comportamiento hacia fines más elevados que los de sus pares? ¿Es posible estudiarlo científicamente?

Mikusinskise determinó a emprender sus estudios doctorales dedicados a los aspectos motivacionales de la personalidad, en el Departamento de Psicología del Instituto de Psiquiatría en el Maudsley Hospital de la Universidad de Londres, dirigida por Hans Eysenck durante el período 1964 a 1966 (Mikusinski, 1973). Se valió de antiguos contactos de la época en que sirvió al ejército británico para lograr la admisión (Mikusinski, 1996). A cinco años de su apertura, el CONICET le otorgó una beca para financiar estos estudios. En estas épocas en que la psicología se abría tímidamente el paso en el sistema científico argentino (Hurtado, 2010), CONICET también le otorgó una beca a la psicóloga experimental Miguelina Guirao para estudiar psicofísica en Harvard (Piñeda, 2016b). En ambos casos, se evidenciaba el apoyo a una psicología científica con énfasis en el estudio de las bases biológicas del comportamiento, sustentada en modelos matemáticos.

\section{Eysenck y la naciente psicología científica sanluiseña}

¿Cómo habría llegado Eva a gestar la idea de estudiar con Eysenck en Londres? Cuando su tesis doctoral fue publicada, gracias al subsidio de la Comisión Asesora de Promoción de la Investigación de la Facultad de Ciencias, Eva -que dedicaba esa obra a su madre y a su hija- agradecía a Horas por haberla introducido al estudio de Eysenck (Mikusinski, 1973). Sin embargo, en el prólogo escrito por el maestro, éste se quitaba el mérito y lo atribuía al clima académico plural e integrador generado en el Instituto ornamentado con los retratos de Freud, Pavlov y Piaget: "Ni el experimento o la estadística, ni la fisiología o el diván, ni la sociología o el inconciente son respuestas absolutas, excluyentes y definitivas" (Horas, 1973, p. 7-8). 
Esa apertura académica extendía el diálogo de la psicología sanluiseña hacia las ciencias naturales y a la matemática que también tenían su lugar en la Facultad de Ciencias. En virtud de ello, la formación estadística como base de la psicometría era sólida, y estaba a cargo del matemático italiano Francisco Murat (Ferrero, lemme Khoury, Todisco, \& Scherman, 2016) que también asesoraba los proyectos de investigación y las prácticas psicométricas (Murat, 1965). Su curso de estadística exigía a los alumnos elaborar un trabajo de investigación que aplicara dichos procedimientos.

Pese a limitaciones materiales similares a otras carreras de psicología del país, esa cultura científica se consolidó desde 1960, con políticas de formación de recursos humanos de posgrado en el exterior, y de promoción de investigación original (Piñeda, 2012).

Este contexto académico fue propicio para que circularan numerosos manuales de psicología en francés, inglés y español que actualizaban al equipo sobre los principales avances europeos y norteamericanos, y constituían las referencias de los cursos que dictaba Horas. En 1958 él inauguraba el dictado de la licenciatura en psicología, presentando la ciencia que tenía por objeto. Eysenck era el autor escogido para que los ingresantes tuvieran una primera comprensión de la materia. Se proponía la lectura crítica de Us et abus de la psychologie, traducción de 1956 (un año antes que la versión española de Biblioteca Nueva) del original en inglés primeramente aparecido en 1953. Esta obra planteaba un panorama sobre los más relevantes problemas sociales que la psicología científica procuraba resolver, buscando concientizar al lector no especializado sobre el modelado que los descubrimientos científicos, particularmente en psicología, ejercen sobre sus vidas. Horas había seleccionado la introducción y los capítulos 9 y 13 que abordaban problemas de la psicología "anormal" (normalidad, sexo y clase social) y de las actitudes sociales (estereotipos nacionales y carácter nacional) (Horas, 1958). El polémico capítulo: "What is wrong with psychoanalysis?", era pasado por alto. En esta oportunidad la selección de textos parecía responder a la necesidad de justificar ante el público local, las bondades de la psicología, como fundamento de la pedagogía y de otras ciencias sociales.

A esos tempranos años, Horas remitía la explicación sobre la inquietud de Eva de realizar una tesis "poco frecuente por su modalidad investigadora en el quehacer psicológico argentino", que se había gestado cuando ella era auxiliar del Instituto, y comenzó "con modestos ensayos sobre las ideas de Robaye y Dembo acerca de los niveles de aspiración que pronto le resultaron insuficientes". Años más tarde, el maestro que esta vez parecía dialogar con psicólogos clínicos, resaltaba que los trabajos de Eysenck, "fundados desde el laboratorio y desde el hospital en criterios factoriales", constituían un 
enfoque clínico, distante del freudiano, "dedicado al individuo como sistema total" (Horas, 1973, p. 7).

En efecto, los registros de los proyectos de investigación conducidos en el Instituto desde 1957, y publicados entre 1960 y 1961, evidencian que varios de sus miembros tenían tempranas noticias de Eysenck y utilizaban este marco referencial para fundamentar investigaciones originales. Algunos artículos (Mikusinski, 1961; Mikusinski, Dagfal, \& Pascual de Fourcade, 1961; Murat, 1960, 1961a, 1961b) documentaban que en el Instituto se leía la versión inglesa de 1947 y de 1955 y la francesa de 1950 de Dimensions of Personality, antes de que apareciera la primera traducción castellana de Scientific Study of Personality de Paidós en 1959. Además, Eysenck fue referencia dela tesis de grado de Mikusinski, dirigida por Murat durante 1963. Allí la tesista propuso un estudio preliminar para elaborar una escala actitudinal para medir la supersticiosidad (Mikusinski, 1964).

En consecuencia, la cultura académica del Instituto configuró oportunidades para que Mikusinski se determinara a realizar sus estudios doctorales bajo la dirección de Eysenck, con el propósito de obtener evidencia experimental de una posible relación entre niveles de aspiración y algunos rasgos tipológicos (Horas, 1973; Mikusinski, 1972).

\section{Mikusinski y Eysenck, un programa de psicología científica en San Luis}

A su regreso de Londres, Mikusinski defendió la segunda tesis doctoral en Psicología de San Luis (Universidad Nacional de Cuyo, n.d.). Horas, reconociendo sus méritos, le cedió el puesto de Directora del Instituto. Desde 1966 desempeñó ese cargo hasta 1972, cuando este cerró, por convertirse la UNC sede San Luis en Universidad Nacional de San Luis.

Desde 1966 en adelante, Mikusinski condujo diversos proyectos de investigación acreditados por la universidad, basados en el modelo eysenckiano. Estos proyectos se articularon con el dictado del curso de Metodología de la Investigación a su cargo, donde enseñaba a investigar con el mayor rigor científico. Las carreras de psicología del país no contaban con las mejores condiciones presupuestarias para montar laboratorios e Institutos (Piñeda, 2012) y la de San Luis no era la excepción. En un antiguo garaje adaptado a las funciones académicas, Eva logró poner en marcha la primera computadora de la Escuela de Psicología, cuyo uso había aprendido en Londres (Andrade \& Leone, 2013). Sus alumnos formulaban problemas, diseñaban y conducían investigaciones, buscando confirmación empírica de postulados de Eysenck y concluyeron un centenar de 
tesis. Algunos se incorporaron al plantel docente con efecto multiplicador (Piñeda, 2010).

Se han conservado en archivo 109 de las tesis logradas entre 1969 y 1983 bajo la dirección de Mikusinski -que representan el $40 \%$ de los egresados del período-, las cuales hemos analizado y clasificado por temas. Registramos que 75 buscaban la verificación empírica de algún aspecto de la teoría de las dimensiones de la personalidad de Eysenck, planteando variedad de problemas: a) estructura motivacional y otras variables: reacción a la frustración, necesidades de filiación, necesidades de agresión, actitudes y expectativas hacia la muerte / suicidio / eutanasia, elección de carrera; b) dimensiones de personalidad y otras variables de personalidad: fuerza del yo, asertividad, ansiedad, control interno y externo, pensamiento y hábitos mentales, creencias sin fundamento; c) dimensiones de personalidad y aspectos biológicos: respuesta fisiológica de salivación (Rodríguez \& Mikusinski, 1977); enfermedades cardiovasculares, dermatológicas, respiratorias, hábito de fumar. Los trabajos incluyeron diversas poblaciones: adolescentes, adultos, madres solteras, ingresantes al servicio militar, cadetes de policía, matrimonios comerciantes, bodegueros, vendedoras de productos de belleza, empleados públicos provinciales, empleados contratados, enfermeras, alumnos, docentes y no docentes, internos de la Colonia Hogar, del Servicio Penitenciario y del Hospital Psiquiátrico.

El resto de las tesis, abordaban algún postulado eysenckiano, pero ponían el acento en la validación de pruebas de otros autores, y se distribuían en cuatro líneas de trabajo.

La primera, integraba aportes de Charles E. Osgood (1916-1991) (Diferencial Semántico) y de Eysenck. Tenía como propósito verificar si la pertenencia a un grupo tipológico determinado sería el responsable de la resonancia afectiva y esquema conductual diferenciales frente a estímulos dados (Omar, n.d.). Esta línea también se vio reflejada en la tesis doctoral que Alicia Omar trabajó durante 1982 titulada: “El Diferencial Semántico como técnica de diagnóstico psicológico y exploración clínica". En 1985 Omar ingresó a CONICET como Investigadora Asistente, dirigida por Mikusinski, iniciando la "Estandarización argentina de los cuestionarios autodescriptivos de personalidad de Eysenck" (Mikusinski, 1986).

Segundo, los trabajos que proponían el estudio del rendimiento en situaciones conflictuales que estimulaban la ansiedad (Mikusinki, 1972; Mikusinki \& Leone, 1981).

La tercera, buscaba relaciones entre la teoría eysenckiana y los estudios motivacionales desde la perspectiva de Robert Kastenbaum (1933-2013) (Kovács, 2008). Esta iniciativa surgió de la experiencia que Mikusinski tuvo con el renombrado tanatólogo en 1972 en la Universidad de Michigan, abonando la idea de un estudio transcultural 
sobre actitudes ante la vejez y la muerte. Además, se logró una adaptación del cuestionario (Mikusinski \& Omar, 1982).

El cuarto grupo de trabajos era sobre percepción social del psicólogo en relación a otros profesionales de la salud como el psiquiatra y el psicoanalista. Estas investigaciones contaban como antecedentes el trabajo de Litvinoff (1970) replicado con una muestra de San Luis por Rodríguez Kauth (1973). Se inscribían en los debates por la legalización del título del psicólogo y las disputas con el campo médico y psicoanalítico por la definición y alcance del rol de psicólogo. Los aportes de Mikusinskise sumaron (Horas, Barbenza, Mikusinski, Montoya, \& Castillo de Pantano, 1977; Mikusinski, Carugno, \& Nassif, 1976) alcanzando visibilidad. Por ejemplo, el Dr. Abraham Luchins, catedrático fundador del Psychology Department at the State University of New York, Albany mostraba interés por estos trabajos (Luchins, 1980).

Entre 1967 y 1969, Mikusinski volvió a incursionar en terreno militar, pero esta vez como psicóloga. Dirigió el proyecto: “Investigación Psicosocial de Ciudadanos de la clase 1947". Consistía en un estudio transversal de la población masculina de la provincia, de 20 años de edad, revisados entre el 20 y el 29 de noviembre de 1967 para ingresar al servicio militar (Mikusinski, 1967). Se ambicionaba extender el estudio a otras regiones del país, aunque no hay constancia de que ello haya ocurrido. Pero, al menos, se captó el interés de CONICET que otorgó financiamiento. Se evaluaron 900 sujetos (Mikusinski, Horas, \& Gialluca, 1969) por parte de docentes y alumnos adscriptos en el Instituto (Mikusinski, Horas, \& Gialluca, 1967).

Se estudiaban rasgos de personalidad, que se consideraban básicos para futuras investigaciones sociológicas, psicológicas y psiquiátricas. Entendemos que se estaba pensando en realizar la primera adaptación del Cuestionario de Personalidad de Eysenck en suelo sudamericano. Se reconocía como antecedente directo el trabajo del Dr. Luis Campoy en Mendoza -que en 1966 había obtenido el auspicio de la Secretaría de Guerra y Secretaría de Salud Pública de la Nación- cuya encuesta psicosocial Mikusinski utilizaba. Integrando los modelos factoriales más influyentes de la época (Boyle et. al, 2016), también aplicaba el Test de inteligencia de R. B. Cattell, el Cuestionario de Personalidad de Eysenck (EPI), que complementaba con la Escala de Angustia Manifiesta de Taylor y el Cuestionario de Creencias Infundamentadas. Profundizando sus estudios de grado, esta era una prueba piloto con la que Mikusinski pretendía apreciar la magnitud de la aceptación de proposiciones de tipo supersticioso y su incidencia en la conducta (Mikusinski, Horas, \& Gialluca, 1967).

En 1976 se creó la Secretaría de Ciencia y Tecnología de la UNSL, que en 1977 acreditó el proyecto dirigido por Mikusinski: "Nuevos intentos de la verificación empírica de la teoría de la personalidad de Eysenck" 
(Omar, n.d.; Omar \& Mikusinski, 1983), también subsidiado por CONICET durante una década (Mikusinski, 1986).

Para 1980 el autor europeo había logrado captar la atención de psicólogos clínicos que buscaban otras perspectivas, más allá de la hegemonía psicoanalítica, y que organizaron en Buenos Aires el Primer Congreso sobre Psicoterapias (Korman, et al., 2015). Héctor Fernández Álvarez, del Instituto de Psicología de la Universidad de Belgrano, presidía la Comisión Organizadora que había invitado a los extranjeros: Hans Eysenck y Rubén Ardila. En ese contexto, también invitó a Mikusinski y a Horas a realizar una presentación en un panel (Fernández Álvarez, 1981). Mikusinski aceptó la invitación para presentar dos trabajos con sus colaboradores (Mikusinski, 1981a), y se puso en contacto con Eysenck para comunicarle el sumo agrado con que había recibido la noticia y la gran expectativa que le generaba su reencuentro (Mikusinski, 1981b).

El científico del Maudsley Hospital obtuvo amplia cobertura de la prensa ya que suscitó acaloradas polémicas por la radical oposición al psicoanálisis que planteaba en tierra dominada por psicoanalistas. Eysenck presentó las bases conceptuales y experimentales de la terapia del comportamiento y argumentó sobre su eficacia, técnicas de seguimiento, evaluación y prevención de recaídas. El evento es recordado como un importante hito en la difusión de la terapia cognitivo-comportamental en Argentina (Korman, et al, 2015).

La otra figura extranjera invitada al congreso, Rubén Ardila, en 1975 fue profesor visitante de la UNSL para el curso Terapia del Comportamiento. Sus vínculos con San Luis habían iniciado en la Sociedad Interamericana de Psicología, de la que Horas había sido Vice-Presidente para América del sur en 1964, y varios docentes de San Luis eran miembros, incluida Mikusinski (Piñeda, 2010).

En 1986, Eva decidió jubilarse para quedar en libertad de acompañar a su hija nombrada Cónsul Adjunto en Francia. Sin embargo, conservaba cierta expectativa de continuar su vida académica. La UNSL la nombró Profesora Consulta y Miembro Representante en Francia, y CONICET la contrató por dos años como Investigadora Principal. Retomó contacto con Paul Fraisse (1911-1996), con quien había trabajado en 1969 con Beca de la Organización de Estados Americanos (Mikusinski, 1972). En esta oportunidad, se incorporó al Laboratorio de Psicología Experimental de La Universidad René Descartes que Fraisse dirigía para conducir un estudio transcultural basado en el modelo eysenckiano, sobre argentinos residentes en Francia (Mikusinski, 1987).

Poco tiempo después, Eva participó en Buenos Aires de la organización de la Sociedad Argentina de Ciencias del Comportamiento (1987), cuya firma consta en el "Acta Fundacional Preliminar" que rubricaron investigadores y docentes de diversas ciencias del comportamiento humano y animal, dedicados 
sistemáticamente a la producción de conocimiento original, básico, aplicado o de desarrollo, desde perspectivas biológicas, psicológicas y sociales sobre bases científicas. Mikusinski recibió propuestas de presidirla, pero declinó (Mikusinski, 1996), siendo elegida Claribel Barbenza.

Al año siguiente, venció su contrato con CONICET, Eva no pidió renovación y cesó su labor académica.

\section{Conclusiones}

Eva Borwowska de Mikusinski, mujer de origen polaco migrada a la Argentina tras la Segunda Guerra Mundial, logró integrarse a la vida académica, sorteando barreras políticas, culturales y económicas, y resolviendo tensiones entre su vida familiar y profesional. El análisis de los auspicios para su integración a la carrera científica (Sprung \& Sprung, 1996), nos muestra que su padre y su abuelo le habilitaron el temprano acceso al conocimiento, y su madre la apoyó en el sostenimiento familiar para lograr su inserción en las instituciones científicas. En el terreno profesional resulta decisivo el auspicio de Plácido Horas, Director de su equipo de trabajo, que la incentivó en sus estudios de grado y de posgrado, así como le cedió su lugar en la Dirección del Instituto de Investigaciones una vez que ella logró su formación doctoral en Londres con Eysenck.

Por otra parte, la feminización de profesiones relativas al trabajo administrativo y educativo (Falcone, 2015; French, 1988) le proveyeron acceso al campo de la psicología. Diversas condiciones institucionales de la UNCy posibilitaron la temprana recepción de Eysenck en San Luis impulsando los estudios de Mikusinski en Londres: pluralidad teórica; circulación de manuales extranjeros originales en inglés o traducciones francesas previas a las que se popularizaron en español en la región; políticas académicas de formación de recursos humanos en el exterior confluyentes con las de CONICET, que promovía el desarrollo de una psicología científica con bases biológicas y matemáticas contrastante con el psicoanálisis predominante en las carreras de psicología (Piñeda \& Scherman, 2016b).

Aportando nuevos datos empíricos a la literatura existente sobre la recepción de Eysenck (Flores-Mendoza et. al., 2016; Korman, et al., 2015; Schmidt, De Costa Oliván, Firpo, Vion, \& Casella, 2008) y de otros modelos factoriales en Sudamérica (Carpintero, 2006; Ferrero, lemme Khorry, Todisco \& Scherman, 2016; Rimoldi, 1995), diversos hechos posicionan a Mikusinski como pionera en la introducción del modelo eysenckiano de evaluación de la personalidad, y demuestran que ella también formaba parte de la comunidad académica local que investigaba integrando diversos modelos factoriales (Rimoldi, 1995). 
Además, entre mediados de 1960 y 1980, Mikusinski se basó en ellos para desarrollar un programa de investigación orientado a problemas locales, que articuló sistemáticamente con la formación de grado y de posgrado de psicólogos, abonando una matriz alternativa a la dominante en Argentina (Dagfal, 2018). Dirigió proyectos de investigación acreditados por la universidad y subsidiados por el CONICET, becarios, un centenar de tesis, varias publicaciones en revistas científicas internacionales; condujo el primer proyecto de adaptación de la Escala de Personalidad de Eysenck en suelo sudamericano; logró uno de los pocos nombramientos de la época en el plantel de CONICET para el área de psicología; fue miembro fundadora de la AACC, miembro de sociedades científicas internacionales, y se vinculaba con académicos norteamericanos y europeos especialistas en estudios de la personalidad. No obstante, el alcance de su obra fue limitado por las crisis políticas y el desfinanciamiento de las carreras de psicología argentinas. Por otra parte, cuando hacia el final de su carrera sus logros académicos se consolidaban y gozaba de reconocimiento de sus pares, se alejó de esta al cumplir su edad de retiro y optar por proyectos familiares. Sin embargo, a los 65 años, estaba dispuesta a volver a empezar.

\section{Referências}

Andrade, M. C., \& Leone, M. E. (2013). La investigación en psicología: Dra. Eva Borkowska de Mikusinski. In R. A. Noir (Ed.), El Bicentenario en la Facultad de Ciencias Humanas. San Luis: Mac Graph.

Ardila, R. (1979). La Psicología en Argentina: pasado, presente, futuro. Revista Latinoamericana de Psicología, 11(1), 71-91. Recuperado de https://www.redalyc. org/pdf/805/80501105. pdf

Arias Gallego, W. L. (2012). Hans Jürgen Eysenck (1916-1997): El Infatigable Investigador de la Personalidad. Revista de Psicología de Trujillo, 14(1), 118-126. Recuperado de revistas.ucv.edu.pe/index.php/R_PSI/article/download/186/98

Asociación Argentina de Ciencias del Comportamiento (1987). Acta Fundacional Preliminar. Sin publicar.

Boyle, G. J., Stankov, L., Martin, N. G., Petrides, K. V., Eysenck, M. W., \& Ortet, G. (2016). Hans J. Eysenck and Raymend B. Cattell on intelligence and personality. Personality and Individual Differences, 103, 40-47. doi: 10.1016/j.paid.2016.04.029

Brock, A. (2014). What is a polycentric History of Psychology? Estudos e Pesquisas em Psicologia, 14(2), 646-659. Recuperado de http://www.epublicacoes.uerj. br/index. php/revispsi/article/view/12565/9749 
Caballo, V. (1997). Hans Jurgen Eysenck (1916-1997). Revista Latinoamericana de Psicología, 29(3), 517-522. Recuperado de www. redalyc.org/pdf/805/80529309.pdf

Carpintero, H., Tortosa, F., \& Sanchis-Aldas, P. (1997). El impacto de $\mathrm{H}$. J. Eysenck en la psicología contemporánea. Revista de Psicología General y Aplicada, 50(4), 417-432. Recuperado de https://dialnet.unirioja.es/descarga/articulo/2365112.pdf

Citlak, A. (2016). The Lvov-Warsaw School: The forgotten tradition of historical psychology. History of Psychology, 19(2), 105-124. doi: $10.1037 /$ hop0000029

Corr, P. J. (2016). Hans J. Eysenck: Introduction to centennial special issue, Personality and Individual Differences, 103, 1-7. doi: 10.1016/j. paid.2016.07.018

Cortada, N. (2006). Autobiografía. Revista de Historia de la Psicología, 27(4), 49-58.

Dagfal, A. (2018). Psychology and Psychoanalysis in Argentina. Politics, French thought and the university connection, 19551976. History of Psychology, 21(3), 254-272. doi: 10.1037/hop0000071

Diener, E., Oishi, S., \& Park, J. Y. (2014). An Incomplete List of Eminent Psychologists of the Modern Era. Archives of Scientific Psychology, 2(1), 20-32. doi: 10.1037/arc0000006

Eysenck, H. J. (1977). Personalidad y sexo en grupo: Un estudio empírico. Revista Latinoamericana de Psicología, 9(1), 21-28. Recuperado de www.redalyc.org/pdf/805/80590103.pdf

Eysenck, M. W. (2016). Hans Eysenck: A research evaluation, Personality and Individual Differences, 103, 209-219. doi: 10.1016/j.paid.2016.04.039

Falcone, R. (2015). Las relaciones sociales de género en la comunidad psicoanalítica argentina: 1942-1960. Revista científica de UCES, 19(1), 90-121. Recuperado de http://dspace.uces.edu.ar:8180/xmlui/bitstream/handle/12345 $6789 / 3316 /$ Relaciones Falcone. pdf?sequence $=1$

Fernández Álvarez, H. (1981). Correspondencia a Eva Mikusinski. Sin publicar.

Ferrero, C., Iemme Khoury, V., Todisco, R., \&, Scherman, P. (2016). Inicios de la investigación experimental en psicología en Córdoba en el periodo 1960-1990. Revista Argentina de Ciencias del Comportamiento, 8(2), 58-66. Recuperado de http: //www.scielo.org.ar/pdf/radcc/v8n2/v8n2a05.pdf

Flores-Mendoza, C., Ardila, R., Gallegos, M., Sampaio Braga, L., Miranda Carvalhais S. B., \& Andrade, M. (2016). Hans Eysenck in Latin America: His influence in the psychology, the study of personality and individual differences. Personality and Individual Differences, 103, 68-73. doi: 10.1016/j. paid.2016.04.049 
French, J. L. (1988). Grandmothers I wish I knew: contributions of women to the history of school psychology. Professional School Psychology, 3(1), 51-68. Recuperado de https://psycnet. apa.org/fulltext/1988-18344-001.pdf

Furumoto, L. (1992). Joining separate spheres - Christine LaddFranklin, woman scientist (1847-1930). American Psychologist, 47(2), 175-182. Recuperado de https://psycnet. apa.org/fulltext/1992-21935-001.pdf

Gottfredson, L. S. (2016). Hans Eysenck's theory of intelligence, and what it reveals about him, Personality and Individual Differences, 103, 116-127. doi:10.1016/j.paid.2016.04.036

Horas, P. (1948). Programa de Psicología I. Facultad de Ciencias de la Universidad Nacional de Cuyo. Sin publicar.

Horas, P. (1973). Prólogo. In E. B. de Mikusinski, Niveles de aspiración y personalidad (Tesis de Doctorado en Psicología). San Luis: Facultad de Pedagogía y Psicología, Universidad Nacional de Cuyo.

Horas, P., Barbenza, C., Mikusinski, E. Montoya, O., \& Castillo de Pantano, J. (1977). La imagen del psicólogo y de la psicología. Acta Psiquiátrica y Psicológica de América Latina, 23(2), 118130.

Hurtado, D. (2010). La ciencia argentina. Un proyecto inconcluso: 1930 - 2000. Buenos Aires: Edhasa.

Jacó-Vilela, A. M., Degani-Carneiro, F. \& Novaes Messias, M. C. (2012). Mujer y psicología: la presencia femenina en la historia de la psicología de Río de Janeiro. Investigaciones en Psicología, 17(2), 11-12.

Klappenbach, H., Marincevic, J., Arias, G., \& Montoya, O. Berasain de (1995). Crónicas de la vida universitaria en San Luis. San Luis:

Editorial Universitaria de San Luis. Recuperado de postgrado.unsl.edu.ar/Cronicas. pdf

Korman, G. P., Viotti, N., Garay, \& C. J. (2015). The origins and professionalization of cognitive psychotherapy in Argentina. History of Psychology, 18(2), 205-214. doi: 10.1037/a0038968

Kovács, M. J. (2008). Desenvolvimento da Tanatologia: estudos sobre a morte e o morrer. Paidéia, 18(41), 457-468. doi: 10.1590/S0103-863X2008000300004

Litvinoff, N. (1970). El psicólogo y su trabajo. Estudio preliminar. Revista Argentina de Psicología, 4, 122-133.

Luchins, A. (1980). Correspondencia a Eva B. Mikusinski. Sin publicar.

Mikusinski, E. (1961). Visión panorámica de estudios recientes sobre teorías de la personalidad. Anales del Instituto de Investigaciones Psicopedagógicas, 6, 305-322.

Mikusinski, E. (1967). Nota al Decano Hugo Arnaldo Fourcade. Sin publicar. 
Mikusinski, E. (1964). Elaboración de una escala actitudinal para medir la supersticiosidad (Estudio preliminar) (Tesis de Licenciatura en Psicología). Universidad Nacional de Cuyo, San Luis.

Mikusinski, E. (1972). Los niveles de aspiración y los niveles de expectación en las condiciones de alta y baja motivación. Revista Latinoamericana de Psicología, 4(3), 287-293. Recuperado de www.redalyc.org/pdf/805/80540301.pdf

Mikusinski, E. (1973). Niveles de aspiración y personalidad. San Luis: Facultad de Pedagogía y Psicología, Universidad Nacional de Cuyo.

Mikusinski, E. (1981a). Correspondencia a H. Fernández Álvarez. Sin publicar.

Mikusinski, E. (1981b). Correspondencia a H. Eysenck. Sin publicar.

Mikusinski, E. (1986). Nota a Carlos Abeledo, Presidente de CONICET. Sin publicar.

Mikusinski, E. (1987). Nota a Decana Esther Picco. Sin publicar.

Mikusinski, E. (1996). Mi largo camino: Testimonio autobiográfico. Cuadernos Argentinos de Historia de la Psicología, 2, 211-238.

Mikusinski, E., Carugno, O., \& Nassif, M. (1976). Imagen del rol del psicólogo clínico y del psiquiatra en la Argentina. Revista Latinoamericana de Psicología, 8(3), 363-374. Recuperado de https: //www.redalyc.org/pdf/805/80580301.pdf

Mikusinski, E., Dagfal, C, \& Pascuale de Fourcade, L. (1961). Exploraciones preliminares de los niveles de aspiración y expectación en adolescentes. Anales del Instituto de Investigaciones Psicopedagógicas, 6, 211-231.

Mikusinski, E, Horas, P., \& Gialluca, D. (1967). Al Señor Decano de la Facultad de Ciencias Prof. Hugo Arnaldo Fourcade. Nota de solicitud de auspicio de proyecto de investigación ante autoridades militares. 6 de septiembre de 1967. Sin publicar.

Mikusinski, E., Horas, P., \& Gialluca, D. (1969). Un programa de investigación psicosocial de la clase 1948 (1). Primer Congreso Nacional de Psicología Social. Salta /Tucumán. Sin publicar.

Mikusinski, E., \& Leone, M. E. (1981). La técnica del dibujo en espejo como elemento diagnóstico en el estudio de la personalidad. Acta Psiquiátrica y Psicológica de América Latina, 27(3), 200208.

Mikusinski, E., \& Omar, A. (1982). La imagen de la vejez explorada mediante el diferencial semántico. Revista Latinoamericana de Psicología, 14(1), 37-53.

Milar, K. S. (2000). The first generation of women psychologists and the psychology of women. American Psychologist, 55(6), 616619.

Mitchell, R. L. C. \& Kumari, V. (2016). Hans Eysenck's interface between the brain and personality: Modern evidence on the 
cognitive neuroscience of personality. Personality and Individual Differences, 103, 74-81. doi:10.1016/j.paid.2016.04.009

Murat, F. (1960). Introducción al análisis factorial. Anales del Instituto de Investigaciones Psicopedagógicas, 5, 212-240.

Murat, F. (1961a). Límites y posibilidades de los cuestionarios autodescriptivos. Anales del Instituto de Investigaciones Psicopedagógicas, 6, 283-303.

Murat, F. (1961b). Los métodos cuantitativos en las ciencias conductuales. Anales del Instituto de Investigaciones Psicopedagógicas, 6, 7-68.

Murat, F. (1965). Estadística aplicada a las ciencias de la conducta. San Luis: Facultad de Ciencias, Universidad Nacional de Cuyo.

Mustaca, A. (2006). La Psicología científica y el análisis del comportamiento en Argentina. Avances en Psicología Latinoamericana, 24, 13-27.

Omar, A. (n.d.). Informe preliminar inédito de la Tesis de Doctorado en Psicología de la Universidad Nacional de San Luis.

Omar, A. (1988). Estandarización argentina de los cuestionarios de personalidad de Eysenck. Revista Chilena de Neuro-Psiquiatría, 42, 83-95.

Omar, A., \& Mikusinski, E. (1983). El grupo sanguíneo como determinante genético de los tipos de personalidad. Revista Interdisciplinaria, 2(4), 153-166.

Ostrovsky, A. E. (2008). La Sociedad de Psicología en Argentina (1908-1913). Treinta y nueve hombres y una mujer. Revista de Historia de la Psicología, 29(2), 55-67.

Papini, M. \& Mustaca, A. (1979). La psicología experimental argentina entre 1956 y1978. Revista Latinoamericana de Psicología, 11(3), 349-361.

Piñeda M. A. (2007). Plácido Alberto Horas, los cursos de Psicología I y II y los inicios de la carrera de psicología en San Luis. Revista de Psicología, 3(5), 89-97.

Piñeda, M. A. (2010). Inicios de la psicología como ciencia del comportamiento en San Luis (Argentina). Revista Argentina de Ciencia del Comportamiento, 2(1), 24-33.

Piñeda, M. A. (2012). Psychology Publications by Professors at Argentine Psychology Programs: 1958-1982. Revista Interamericana de Psicología/Interamerican Journal of Psychology, 46(1), 111-122.

Piñeda, M. A. \& Jacó-Vilela, M. A. (2014). Ciencia psicológica y profesionalización en Argentina y Brasil: 1930-1980. Universitas Psychologica, 13(5), 2015-2033

Piñeda, M. A. \& Scherman, P. (2016a). Estudios experimentales de la percepción desde la profesionalización de la psicología en Argentina. Estudos e Pesquisas em Psicologia, 16(1), 295-316. 
Piñeda, M. A. \& Scherman, P. (2016b). S. S. Stevens, M. Guirao y los estudios psicofísicos en Argentina. Revista Mexicana de Análisis de la Conducta, 42(2), 153-178.

Pinillos, J. L. (1997). Conversaciones con Hans Eysenck. Revista de Psicología General y Aplicada, 50(4), 409-416. Recuperado de https: // dialnet. unirioja.es/servlet/articulo? codigo $=2365110$

Radtke, H. L., Hunter, M., Stam, H. J. (2000). In memoriam as in life: gender and psychology in the obituaries of eminent psychologists. Canadian Psychology, 41(4), 213-229.

Rimoldi, H. J. A. (1995). Testimonio autobiográfico. Cuadernos Argentinos de Historia de la Psicología, 1(1/2), 275-300.

Rodríguez, J., \& Mikusinski, E. (1977). La salivación como medida fisiológica de la dimensión introversión - extraversión. Revista Latinoamericana de Psicología, 9(2), 201-211.

Rodríguez Kauth, A. (1973). Estado actual de la situación del psicólogo en San Luis. Revista de Psicología (en línea), 6, 105115. http//www.fuentesmemoria.fahce.unlp.edu.ar/art_revistas/pr. 8 98.pdf

Schmidt, V., De Costa Oliván, M. E., Firpo, L., Vion, D., \& Casellahans, L. (2008). Jürgen Eysenck. Vida y obra de uno de los más influyentes científicos de la historia de la psicología. Avances en Psicología Latinoamericana, 26(2), 304-317. Recuperado https://revistas.urosario.edu.co/index. php/apl/article/view/69

Sprung, H. \& Sprung, L. (1996). Women in Psychological History. Forms of integration in Psychology and Women's lecturing activities at German Psychology Congresses: 1904-1978. Revista de Historia de la Psicología, 17(1/2), 185-202.

Universidad Nacional de Cuyo (n.d.). Libro de Egresados. Sin publicar.

Winckler Müller, M. I., \& Reyes, M. I. (2015). Historias de mujeres en la Psicología Chilena: contribuciones de Lola Hoffmann, Hélliette Saint Jean y Vera Kardonsky. Psykhe, 24(1), 1-11. doi: 10.7764/psykhe.24.1.658

\section{Endereço para correspondência \\ María Andrea Piñeda}

Universidad Nacional de San Luis

Consejo Nacional de Investigaciones Científicas y Técnicas, Facultad de Psicología

Ejército de los Andes 950, Edificio Plácido Horas, $1^{\circ}$ piso, oficina 59, CP 5700 , San Luis, Argentina

Endereço eletrônico: mapineda@unsl.edu.ar 
Recebido em: 11/09/2018

Reformulado em: 20/11/2018

Aceito em: 30/11/2018

\section{Notas}

* Doctora en Psicología. Investigadora Adjunta del Consejo Nacional de Investigaciones Científicas y Técnicas. Profesora Adjunta de la Facultad de Psicología de la Universidad Nacional de San Luis donde dirige el Museo de Historia de la Psicología.

**Esta investigación fue realizada con el financiamiento del Consejo Nacional de Investigaciones Científicas y Técnicas (CONICET) y de la Universidad Nacional de San Luis (UNSL).

Este artigo de revista Estudos e Pesquisas em Psicologia é licenciado sob uma Licença Creative Commons Atribuição-Não Comercial 3.0 Não Adaptada. 